\title{
Use of UpToDate and Outcomes in US Hospitals
}

\author{
Thomas Isaac, MD, MBA, MPH'1 Jie Zheng, $\mathrm{PhD}^{2}$, Ashish Jha, MD, MPH $\mathrm{H}^{2,3,4 *}$
}

${ }^{1}$ Division of General Internal Medicine and Primary Care, Beth Israel Deaconess Medical Center, Boston, Massachusetts; ${ }^{2}$ Department of Health Policy and Management, Harvard School of Public Health, Boston, Massachusetts; 'Bivision of General Medicine, Brigham and Women's Hospital, Boston, Massachusetts; ' $V$ A Boston Healthcare System, Boston, Massachusetts

BACKGROUND: Computerized clinical knowledge management systems hold enormous potential for improving quality and efficiency. However, their impact on clinical practice is not well known.

OBJECTIVE: To examine the impact of UpToDate on outcomes of care.

DESIGN: Retrospective study.

SETTING: National sample of US inpatient hospitals.

PATIENTS: Fee-for-service Medicare beneficiaries.

INTERVENTION: Adoption of UpToDate in US hospitals.

MEASUREMENT: Risk-adjusted lengths of stay, mortality rates, and quality performance.

RESULTS: We found that patients admitted to hospitals using UpToDate had shorter lengths of stay than patients admitted to non-UpToDate hospitals overall (5.6 days vs 5.7 days; $P<0.001$ ) and among 6 prespecified conditions (range, -0.1 to -0.3 days; $P<0.001$ for each). Further, patients admitted to UpToDate hospitals had lower riskadjusted mortality rate for 3 of the 6 conditions (range, $-0.1 \%$ to $-0.6 \%$ mortality reduction; $P<0.05$ ). Finally, hospitals with UpToDate had better quality performance for every condition on the Hospital Quality Alliance metrics. In subgroup analyses, we found that it was the smaller hospitals and the non-teaching hospitals where the benefits of the UpToDate seemed most pronounced, compared to the larger, teaching institutions where the benefits of UpToDate seemed small or nonexistent.

CONCLUSIONS: We found a very small but consistent association between use of UpToDate and reduced length of stay, lower risk-adjusted mortality rates, and better quality performance, at least in the smaller, non-teaching institutions. These findings may suggest that computerized tools such as UpToDate could be helpful in improving care. Journal of Hospital Medicine 2012;7:85-90 (C 2011 Society of Hospital Medicine
New health information technologies hold enormous potential for improving the quality and efficiency of healthcare. One commonly used health information technology is computerized clinical knowledge management (CKM) systems, which provide clinicians with access to relevant and continually updated clinical information about major medical topics at the point of care. Studies indicate that clinicians often have questions about patient care, which go largely unanswered during patient encounters. ${ }^{1-3}$ The availability of answers to critical clinical questions can have a large impact on clinical decision-making and practice. $^{4}$

UpToDate is one of the most widely used computerized clinical knowledge management systems in the nation. ${ }^{1,2,5,6}$ Previous studies of UpToDate and similar systems demonstrated that these systems improve

\footnotetext{
*Address for correspondence and reprint requests: Ashish Jha, Harvard School of Public Health, 677 Huntington Ave, Boston, MA 02115; Telephone: 617-432-5551; Fax: 617-432-4494; E-mail: ajha@hsph. harvard.edu

Additional Supporting Information may be found in the online version of this article.

Received: October 25, 2010; Revised: May 2, 2010; Accepted: May 11, 2011

2011 Society of Hospital Medicine DOI 10.1002/jhm.944

Published online in Wiley Online Library (Wileyonlinelibrary.com).
}

acquisition of knowledge, increase the number of answered clinical questions, and change management decisions. ${ }^{7,8}$ However, whether these changes lead to real improvements in clinical outcomes is unknown. Given the urgent need to improve both the quality and efficiency of healthcare, understanding whether UpToDate has the potential to improve outcomes is critical.

Therefore, we examined whether the use of UpToDate was associated with lower risk-adjusted mortality rates, shorter lengths of stay, and better performance on standard quality process metrics. Further, we sought to determine whether the impact of UpToDate was particularly potent in certain subsets of hospitals. Finally, we examined whether the duration of use of UpToDate was associated with better outcomes.

\section{METHODS \\ Overview}

Our overall approach was to examine the relationship between UpToDate and 3 main outcomes: riskadjusted length of stay, risk-adjusted mortality, and performance on standard quality process metrics in the period from 2004 to 2006 . We took 4 approaches to try to reduce potential confounding (adopters of the UpToDate are likely different than non-adopters). First, we used a longitudinal modeling approach 
where hospitals were allowed to serve as their own controls over time. For example, if a hospital was an adopter of UpToDate in the fourth quarter of 2005, all data for that hospital prior to that quarter of 2005 would be counted as part of the control hospitals. Second, we used multivariable models to adjust for observable differences between adopters and nonadopters. Third, we tested for interactions to see if the effect of UpToDate was particularly concentrated in a subset of hospitals. Finally, we examined whether the duration of use, which reflects a potential "doseresponse" relationship, was related to the outcomes of interest.

\section{UpToDate}

The UpToDate system provides a compendium of regularly revised, evidence-based monographs on topics in adult internal medicine, pediatrics, and obstetrics and gynecology. ${ }^{9}$ The system is available through multiple medias (ie, the Internet, handheld devices). Providers at subscribing hospitals can usually access it through any computer terminal within the facility, and often through remote access.

\section{Data Sources and Linkage}

We used 5 primary sources of data: UpToDate usage data, which was provided directly by UpToDate and includes a list of all hospitals in the United States who use the UpToDate software and the start date of usage; the American Hospital Association (AHA) Annual Survey, which contains individual hospital structural characteristics (such as size, location, teaching status); the 2007 Medicare Inpatient Impact files, which includes hospital characteristics not available in the AHA data; the 2004-2006 Medicare Provider Analysis and Review (MEDPAR) databases, which have patient-level discharge information about all Medicare fee-for-service patients hospitalized in a given year; and the 2004-2007 Hospital Quality Alliance (HQA) database, which includes publicly available data for inpatient quality measures. We linked these 4 datasets with a database of hospitals that use UpToDate.

\section{Outcomes}

We chose, a priori, to examine 3 primary outcomes: risk-adjusted length of stay (LOS), which is considered a measure of efficiency; risk-adjusted mortality, a commonly used marker of quality of care; and performance outcomes on HQA quality metrics.

\section{Risk-Adjusted LOS and Risk-Adjusted Mortality Rates}

We examined risk-adjusted LOS and risk-adjusted mortality rates among all hospitalized patients and among 6 common medical and surgical conditions: acute myocardial infarction (AMI), congestive heart failure (CHF), pneumonia, gastrointestinal hemorrhage, stroke, and hip fracture. We selected these 6 conditions because they are used by the Agency for Healthcare Research and Quality (AHRQ) to measure hospital quality. We used International Classification of Diseases, Ninth Revision (ICD-9) codes used by the AHRQ Inpatient Quality Indicators to identify patients admitted for these 6 conditions. ${ }^{10}$ We performed risk-adjustment using the Elixhauser comorbidity adjustment scheme, which was developed by AHRQ and is commonly used to adjust for differences in severity using administrative data.

\section{Quality Processes of Care}

To examine hospital quality performance, we used the HQA process measures for 4 conditions from 2004 to 2007: AMI, CHF, pneumonia, and surgical infection prevention (SIP). We examined all HQA indicators publically available in 2004 (8 measures for AMI, 4 measures for CHF, 6 measures for pneumonia, and 2 measures for SIP). (The specific indicators are listed in Supporting Appendix Table 1 in the online version of this article.) We created summary scores for each condition, and an overall hospital summary score for the performance on all indicators, using methodology previously described by the Joint Commission. ${ }^{11}$ Each summary score represents the number of times a hospital performed the appropriate action across all measures for that condition divided by the number of "opportunities" the hospital had to provide appropriate care for that condition. Composite scores were only calculated if a hospital had at least 30 patients for at least one of the measures of each condition.

\section{Analysis-CKM Users Versus Non-Users}

We chose, a priori, to perform several sets of analyses to understand the relationship between the use of UpToDate and clinical outcomes. Our primary approach was a longitudinal model where each hospital was allowed to serve as its own control. In sensitivity analyses, we used a "differences-in-differences" model where we examined whether the changes for hospitals that adopted UpToDate differed compared to changes in outcomes for non-adopters, adjusting for temporal trends by using time as a covariate in the model.

In the first analysis using longitudinal data, we examined whether being admitted to a hospital with UpToDate was associated with shorter length of stay, lower risk-adjusted 30-day mortality or higher process quality. This model allowed each hospital to serve as its own control and tested to see how the outcomes changed after adoption of UpToDate, controlling for secular trends by including non-UpToDate hospitals. The models were adjusted for hospital characteristics including size, region, location (urban vs rural), ownership (for-profit, not-for-profit private, not-for-profit public), teaching status (member of the Council of Teaching Hospital vs not), the proportion of patients that had Medicaid, the Disproportionate Share 
TABLE 1. Characteristics of Hospitals Using and Not Using UpToDate

\begin{tabular}{lccc}
\hline \multicolumn{1}{c}{ Characteristics } & $\begin{array}{c}\text { Using UpToDate } \\
(\mathrm{N}=1017)\end{array}$ & $\begin{array}{c}\text { Not Using UpToDate } \\
(\mathrm{N}=2305)\end{array}$ & $P$ Value \\
\hline Hospital size & $\%$ & $\%$ & $<0.001$ \\
Small (6-99) & 13 & 36 & \\
Medium (100-399) & 64 & 55 & $<0.001$ \\
Large (400+) & 23 & 8 & \\
Hospital region & & & \\
Northeast & 27 & 12 & \\
Midwest & 26 & 23 & $<0.001$ \\
South & 27 & 47 & \\
West & 20 & 18 & \\
Profit status & & & \\
Profit hospitals & 10 & 21 & $<0.001$ \\
Nongovernment nonprofit & 75 & 61 & \\
Government nonprofit & 15 & 18 & \\
Teaching hospitals & 19 & 4 & \\
Urban location & 96 & & \\
\hline
\end{tabular}

Hospital (DSH) Index, and the presence or absence of a medical intensive care unit (ICU). We used a repeated-measures generalized estimating equations (GEE) to account for both clustering at the hospital level and for the repeated measures nature of our analysis. We used the Elixhauser comorbidity adjustment scheme to account for patient-level factors. ${ }^{12}$

In our first set of models, we included all patients. We subsequently built 6 condition-specific models for each of the 6 common medical conditions: AMI, CHF, pneumonia, gastrointestinal hemorrhage, stroke, and hip fracture.

\section{Identifying Subsets of Hospitals}

Next, we postulated a priori that certain subsets of hospitals-smaller institutions and non-teaching institutions-might have less access to high-quality clinical information and, thus, be mode likely to benefit from UpToDate. To determine if the potential impact of UpToDate on the outcomes varied based on these hospital characteristics, we repeated our analyses using multivariable models but tested interaction terms. We found significant interactions for 1 outcome (HQA quality performance scores), and present data with stratified analyses for this outcome.

\section{Impact of Duration of Use}

Finally, we calculated duration of UpToDate use for each hospital. For each hospital using UpToDate, we identified the date it started using the system. Based on the start date, we calculated each hospital's duration of use for each quarter. We used the midpoint of that quarter to calculate the number of days a hospital used UpToDate. For example, if a hospital started using UpToDate on January 1, 2002, we assigned 775 days for its duration of use for the first quarter of 2004 (365 days per year $\times 2$ years +45 days for the first quarter of 2004) and 865 days for its duration of
TABLE 2. Risk-Adjusted Length of Stay for Hospitals Using UpToDate Compared to Non-Users

\begin{tabular}{lllcr}
\hline Conditions & $\begin{array}{c}\text { Using } \\
\text { UpToDate (Days) }\end{array}$ & $\begin{array}{c}\text { Not Using } \\
\text { UpToDate (Days) }\end{array}$ & $\begin{array}{c}\text { Difference } \\
\text { (Cl) (Days) }\end{array}$ & \multicolumn{1}{c}{$P$ Value } \\
\hline Total & 5.6 & 5.7 & $-0.1(-0.2$ to -0.0$)$ & 0.001 \\
AMl & 5.3 & 5.5 & $-0.2(-0.3$ to -0.2$)$ & $<0.001$ \\
CHF & 5.6 & 5.7 & $-0.2(-0.2$ to -0.1$)$ & $<0.001$ \\
PN & 6.3 & 6.5 & $-0.2(-0.2$ to -0.1$)$ & $<0.001$ \\
Stroke & 5.9 & 6.0 & $-0.1(-0.2$ to -0.1$)$ & $<0.001$ \\
GIH & 5.3 & 5.4 & $-0.2(-0.3$ to -0.2$)$ & $<0.001$ \\
Hip fracture & 6.7 & 6.8 & $-0.1(-0.2$ to -0.1$)$ & $<0.001$
\end{tabular}

NOTE: Quarterly data from 2004 through 2006. All analyses are adjusted for hospital characteristics including size, census region, urban vs rural location, ownership (for-profit, not-for-profit private, not-for-profit public), teaching status (member of the Council of Teaching Hospital vs not), and the presence or absence of a medical intensive care unit (ICU). Analyses were also adjusted for patient-level factors and comorbidites using methodology developed by Elixhauser.

Abbreviations: AMl, acute myocardial infarction; $\mathrm{CHF}$, congestive heart failure; $\mathrm{Cl}$, confidence interval; $\mathrm{GH}$, gastrointestinal hemorrhage; PN, pneumonia.

use for the second quarter of 2004. We excluded hospitals that did not use UpToDate.

Our primary analysis used a dataset that was restricted to just those hospitals using UpToDate. We ran the model for all discharges and for each of the 6 conditions for both LOS and for risk-adjusted mortality rate, as well as for the 4 conditions encompassed in the HQA quality reporting program. In all analyses, we considered a 2 -sided $P$ value as statistically significant.

\section{Assessing the Overall Impact of UpToDate}

To better assess the clinical significance of any change in mortality rates, we calculated the number of deaths prevented if all hospitals saw the same gain in mortality if they adopted UpToDate. To calculate this impact number, we identified the overall reduction in risk-adjusted mortality associated with UpToDate adoption and multiplied this number by the number of elderly Medicare patients admitted to non-UpToDate hospitals. We calculated a similar number for changes in length of stay.

\section{RESULTS}

We found that between 2004 through 2006, 1017 hospitals used UpToDate for at least 1 quarter. Users of UpToDate were more likely to be large, urban, teaching hospitals located in the Northeast, and either public or nonprofit (private) hospitals (Table 1).

Over the 3 years, patients admitted to hospitals with UpToDate had generally shorter lengths of stay for all hospitalizations than patients admitted to hospitals without this specific system (5.6 days vs 5.7 days, $P<$ 0.001 ; Table 2), and shorter lengths of stay for each of the 6 conditions examined ( 0.1 to 0.3 days shorter LOS, $P<0.001$; Table 2).

Similarly, we found that hospitals with UpToDate had lower risk-adjusted 30-day mortality rates, although the effects here were less consistent (Table $3)$. When we examined individual conditions, we found that patients admitted to UpToDate hospitals 
TABLE 3. Risk-Adjusted 30-Day Mortality Rates Among Hospitals Using UpToDate Compared to Non-Users

\begin{tabular}{lcccc}
\hline Conditions & $\begin{array}{c}\text { Using } \\
\text { UpToDate (\%) }\end{array}$ & $\begin{array}{c}\text { Not Using } \\
\text { UpToDate }(\%)\end{array}$ & $\begin{array}{c}\text { \% Difference } \\
(\mathrm{Cl})\end{array}$ & $P$ Value \\
\hline Total & 9.0 & 9.1 & $-0.1(-0.2$ to 0.0) & 0.04 \\
AMl & 18.4 & 19.0 & $-0.7(-1.2$ to -0.2$)$ & 0.03 \\
CHF & 11.1 & 11.3 & $-0.2(-0.4$ to -0.1$)$ & 0.21 \\
PN & 12.1 & 12.6 & $-0.5(-0.7$ to -0.2$)$ & $<0.001$ \\
Stroke & 19.9 & 19.9 & $-0.02(-0.5$ to 0.5$)$ & 0.91 \\
GlH & 6.9 & 7.3 & $-0.4(-0.7$ to -0.2$)$ & 0.001 \\
Hip fracture & 8.8 & 8.6 & $0.2(-0.2$ to 0.5) & 0.41 \\
\hline
\end{tabular}

NOTE: Rates from 2004 through 2006. All analyses are adjusted for hospital characteristics and patient characteristics.

Abbreviations: $\mathrm{AMl}$, acute myocardial infarction; $\mathrm{CHF}$, congestive heart failure; $\mathrm{Cl}$, confidence interval; $\mathrm{GlH}$, gastrointestinal hemorrhage; PN, pneumonia.

had lower risk-adjusted 30-day mortality rate for 4 of the 6 conditions, although only 3 of those 4 differences were statistically significant (Table 3 ). For example, patients in UpToDate hospitals had a lower likelihood of mortality for AMI $(18.4 \%$ vs $18.9 \%, P=$ $0.03)$.

We found a more consistent association between the adoption of UpToDate and quality performance. Hospitals that had UpToDate had higher performance for each of the 4 conditions examined. For example, hospitals with UpToDate had higher quality performance for AMI compared to hospitals that did not adopt UpToDate $(93.2 \%$ vs $90.4 \%, P<0.001$; Table 4). The results for CHF, pneumonia, and surgical complication prevention were qualitatively similar, and each reached statistical significance (Table 4).

In analyses that test for interaction, we found that the relationship between UpToDate use and quality performance was modified by hospital size and teaching status. Specifically, much of the benefit of UpToDate seemed limited to small and medium-sized hospitals, as well as non-teaching hospitals. For example, among small hospitals, those with UpToDate had, on average, 3-7 point greater performance on the HQA scores, but almost no effect was found among large hospitals. Similarly, we found that non-teaching hospitals were likely to have better performance in each of these areas if they had UpToDate, but this relationship was not consistent among major teaching hospitals (Table 5).

In our analyses of duration of use of UpToDate, we found a consistent relationship with shorter lengths of stay (see Supporting Appendix Table 2 in the online version of this article). Each 1000 days of UpToDate use was associated with a 0.08 day shorter length of stay $(P<0.001)$. A similar relationship was present and statistically significant in each of the 6 conditions examined. When we examined the impact of duration of use of UpToDate on risk-adjusted mortality rate, we found a comparable relationship: Duration was associated with a lower mortality rate overall and for
TABLE 4. UpToDate Use and Performance on the Standard Quality Indicators

\begin{tabular}{cllcr}
\hline \multicolumn{1}{c}{ Conditions } & $\begin{array}{c}\text { Using } \\
\text { UpToDate }(\%)\end{array}$ & $\begin{array}{c}\text { Not Using } \\
\text { UpToDate }(\%)\end{array}$ & $\begin{array}{c}\text { \% Difference } \\
(\mathrm{Cl})\end{array}$ & P Value \\
\hline AMl summary score & 93.4 & 90.2 & $3.2(2.6,3.6)$ & $<0.001$ \\
CHF summary score & 81.0 & 75.1 & $5.9(5.0,6.8)$ & $<0.001$ \\
PN summary score & 83.7 & 83.1 & $0.6(0.3,0.9)$ & 0.003 \\
SIP summary score & 80.0 & 78.1 & $1.9(1.0,2.9)$ & 0.002 \\
\hline
\end{tabular}

NOTE: All analyses are adjusted for hospital characteristics and patient characteristics. Data are based on performance on the Hospital Quality Alliance (HQA) indicators; UpToDate use and HQA scores among all hospitals, 2004 through 2007.

Abbreviations: AMI, acute myocardial infarction; $\mathrm{CHF}$, congestive heart failure; $\mathrm{Cl}$, confidence interval; $\mathrm{PN}$, pneumonia, SIP, surgical infection prevention.

5 of the 6 conditions examined, although only significant for 3 of the conditions (see Supporting Appendix Table 2 in the online version of this article). Similarly, greater duration was associated with better quality performance for each of the 4 conditions in the HQA program (data not shown).

When we quantified the overall impact of UpToDate, we found that if non-adopters had a similar benefit in mortality $(0.1 \%)$ seen in hospitals that adopted this system, it would lead, overall, to approximately 5550 fewer deaths annually $(95 \%$ confidence interval 2601 fewer deaths to 7529 fewer deaths) and the 0.1 days shorter length of stay would lead to approximately 523,000 fewer patient days $(95 \%$ confidence interval 160,000 to 799,000 fewer days) out of the approximately 30 million patient-days that occurred in 2006 among non-UpToDate hospitals.

\section{DISCUSSION/CONCLUSION}

We found that use of a commonly used computerized clinical knowledge management system (UpToDate) was associated with consistent, although small, reductions in lengths of stay, lower risk-adjusted mortality rates, and higher quality performance. Much of the quality performance benefit seemed to be limited to small and medium-sized, non-teaching hospitals, while larger teaching hospitals realized little benefit. We found a stronger relationship between duration of use and better outcomes among UpToDate hospitals. Our findings suggest that hospitals using UpToDate had modestly better care that was also somewhat more efficient.

Prior studies have demonstrated that clinical decision support tools (ie, drug-drug alerts and electronic reminders) can improve processes of care and enhance quality. ${ }^{13}$ Computerized clinical knowledge management systems, such as UpToDate, have unique advantages over other computerized clinical decision support tools. For example, computerized clinical knowledge management systems generally do not require electronic health records and can provide guidance to clinicians over a broader spectrum of diseases and clinical scenarios. UpToDate has previously shown to help providers answer questions rapidly, 
TABLE 5. UpToDate Use and HQA Scores, Stratified by Size and Teaching Status, 2004-2007

\begin{tabular}{|c|c|c|c|c|c|}
\hline & & Using UpToDate (\%) & Not Using UpToDate (\%) & \% Difference $(\mathrm{Cl})$ & $P$ Value \\
\hline \multirow[t]{12}{*}{ Hospital size } & Small (6-99 beds) & & & & \\
\hline & AMl summary score & 90.3 & 87.9 & $2.35(0.91,3.78)$ & $<0.001$ \\
\hline & CHF summary score & 75.7 & 69.6 & $6.11(4.04,8.18)$ & $<0.001$ \\
\hline & PN summary score & 84.9 & 83.2 & $1.67(0.83,2.51)$ & $<0.001$ \\
\hline & Medium (100-399 beds) & & & & \\
\hline & AMl summary score & 93.0 & 90.5 & $2.52(2.04,3.00)$ & $<0.001$ \\
\hline & CHF summary score & 81.1 & 78.9 & $2.22(1.31,3.12)$ & $<0.001$ \\
\hline & PN summary score & 84.0 & 83.2 & $0.84(0.31,1.36)$ & $<0.001$ \\
\hline & Large (>399 beds) & & & & \\
\hline & AMl summary score & 94.7 & 93.8 & $0.82(0.19,1.46)$ & $<0.001$ \\
\hline & CHF summary score & 82.9 & 82.7 & $0.21(-1.27,1.69)$ & 0.83 \\
\hline & PN summary score & 82.4 & 82.7 & $-0.34(-1.49,0.81)$ & 0.39 \\
\hline \multirow[t]{8}{*}{ Teaching status } & Major teaching & & & & \\
\hline & AMl summary score & 94.9 & 94.8 & $0.15(-0.62,0.92)$ & 0.60 \\
\hline & CHF summary score & 83.3 & 83.0 & $0.26(-1.73,2.25)$ & 0.83 \\
\hline & PN summary score & 81.7 & 81.7 & $0.00(-1.67,1.67)$ & 0.95 \\
\hline & Not major teaching & & & & \\
\hline & AMl summary score & 92.7 & 90.1 & $2.59(2.15,3.02)$ & $<0.001$ \\
\hline & CHF summary score & 80.1 & 75.0 & $5.04(4.18,5.90)$ & $<0.001$ \\
\hline & PN summary score & 84.3 & 83.2 & $1.05(0.64,1.47)$ & $<0.001$ \\
\hline
\end{tabular}

NOTE: All analyses are adjusted for hospital characteristics and patient characteristics.

Abbreviations: AMI, acute myocardial infarction; CHF, congestive heart failure; $\mathrm{Cl}$, confidence interval; HQA, Hospital Quality Alliance; PN, pneumonia.

which can lead to changes in decision-making that can improve management and efficiency. 1,7,14

Ours is the first national study, to our knowledge, that has directly examined the relationship between UpToDate and key outcome metrics. Bonis et al. have previously examined the use of UpToDate and its relationship to risk-adjusted LOS and mortality in a limited set of hospitals using a proprietary risk-adjustment scheme. ${ }^{15}$ They found similar results among the "Thompson 100" hospitals that were clinical knowledge management system users (compared to nonusers), including modestly shorter lengths of stay and a trend towards lower mortality. Our findings build on this work, but use publicly available data, a national sample, a well-validated risk-adjustment approach, and a much longer time period. The consistency of the findings across the 2 studies, despite differences in the approaches, help lend confidence that the results are unlikely to be due to chance alone.

There are likely to be important considerations surrounding the costs of UpToDate systems and whether those who purchase the system are "wealthier" than hospitals that chose not to. The typical annual subscription costs for a 100-bed hospital between 2006 and 2010 was $\$ 10,578$, which likely represents less than $0.01 \%$ of the annual operating costs for a 100 bed institution (and is approximately the amount Medicare reimbursed for a single case of pneumonia without complications). Whether this cost would prohibit the adoption of UpToDate for most hospitals is unclear, and it is possible that a hospital that spent nearly $\$ 10,600$ a year in such a system might therefore forego other quality improvement efforts. We suspect that these effects likely vary from hospital to hospital.
The primary limitation of the study is our inability to address whether or not the associations we found between UpToDate and outcomes are causally related. This is a fundamental limitation of all nonrandomized data. Four factors should lend some confidence to the interpretation that these findings may not be due to confounding alone. First, the effects were consistent across a series of measures (mortality, efficiency, and processes) that are not, themselves, highly correlated with each other ${ }^{16-18}$. Our findings, that hospitals with UpToDate were somewhat better across all measures examined, point to the potential benefit of having high-quality clinical information readily available for clinicians. Second, we found that the benefits persisted even after controlling for other hospital characteristics that were associated with adoption, including measures of hospital financial health (as measured by proportion of Medicaid patients and the DSH Index). Of course, this does not negate the possibility that other factors, such as the presence of medical libraries or a culture of quality and continuous learning, may be associated both with the use of UpToDate and with the outcomes. Third, the effects, at least for quality performance, were prominent among smaller, nonteaching hospitals (which, one would surmise, a priori, to be most likely to benefit from UpToDate). Finally, the dose-response relationship of duration of use, which was an analysis limited to only those hospitals with UpToDate, provides more evidence that the system itself may have some impact.

Another limitation of our work is that we used administrative data for risk-adjustment, which has inherent challenges. ${ }^{19-25}$ Given that users of UpToDate, such as teaching hospitals and larger institutions, generally have a much sicker patient population, 
inadequate risk-adjustment may have lead us to underestimate the true effect. It is also possible that many of the hospitals designated as "non-users" had other clinical knowledge management systems of which we were unaware. However, this would likely have made it harder to find an effect, biasing our study towards a null finding. We conducted a series of analyses and, yet, did not adjust for "multiple" testing. We had chosen these analyses a priori and, although the association we found may have been due to selection bias, it is unlikely that all of the associations in our analyses were due to random chance. Next, although we examined the potential impact of UpToDate, we suspect that any high-quality clinical knowledge management system should allow clinicians to deliver higher quality, more efficient care. Finally, we are unsure whether or not the magnitude of effect we found is clinically significant. While the added advantage of having UpToDate appeared to be a reduction in mortality of only $0.1 \%$ (over all conditions), such a difference would be associated with approximately 5550 fewer deaths each year among Medicare fee-for-service beneficiaries. Whether such a benefit would be worth the cost of implementing systems like UpToDate needs to be further explored.

In conclusion, we found consistent association between use of a widely deployed computerized clinical knowledge management system, UpToDate, and reduced length of stay, lower risk-adjusted mortality rates, and higher quality performance. Whether use of UpToDate led to better care is not definitive, but our findings suggest that these types of management software may play an important role as our nation endeavors to improve the quality and efficiency of the healthcare system.

Disclosures: This study was funded by UpToDate, Inc. The funder had no role in study design, input into analyses presented, drafting or editing the manuscript, nor saw the manuscript prior to submission.

All coauthors have seen and agree with the contents of the manuscript. Drs. Jha and Isaac jointly wrote all drafts of the manuscript with no input from any outside sources. Dr. Zheng reviewed the Methods section and provided editorial comments on all sections of the paper.

\section{REFERENCES}

1. Ely JW, Osheroff JA, Chambliss ML, Ebell MH, Rosenbaum ME. Answering physicians' clinical questions: obstacles and potential solutions. I Am Med Inform Assoc. 2005;12(2):217-224.

2. Ely JW, Osheroff JA, Ebell MH, et al. Obstacles to answering doctors' questions about patient care with evidence: qualitative study. BMJ. 2002;324(7339):710.
3. Green ML, Ruff TR. Why do residents fail to answer their clinical questions? A qualitative study of barriers to practicing evidence-based medicine. Acad Med. 2005;80(2):176-182.

4. Swinglehurst DA, Pierce M, Fuller JC. A clinical informaticist to support primary care decision making. Qual Health Care. 2001;10(4): 245-249.

5. Shariff SZ, Bejaimal SA, Sontrop JM, et al. Searching for medical information online: a survey of Canadian nephrologists. J Nephrol. Feb 23, 2011. doi: 10.5301/JN.2011.6373.

6. Hoogendam A, Stalenhoef AFH, de Vries Robbé PF, Overbeke AJPM. Answers to questions posed during daily patient care are more likely to be answered by UpToDate than PubMed. J Med Internet Res 2008;10(4):e29.

7. Lucas BP, Evans AT, Reilly BM, et al. The impact of evidence on physicians' inpatient treatment decisions. J Gen Intern Med. 2004; $19(5$ pt 1):402-409.

8. Blackman D, Cifu A, Levinson W. Can an electronic database help busy physicians answer clinical questions? [abstract]. J Gen Intern Med. 2002;17(suppl 1):220.

9. Fox GN, Moawad N. UpToDate: a comprehensive clinical database. J Fam Pract. 2003;52(9):706-710.

10. AHRQ Quality Indicators. Guide to Inpatient Quality Indicators: Quality of Care in Hospitals - Volume, Mortality, and Utilization [Version 3.1]. Rockville, MD: AHRQ; Mar 12, 2007.

11. Kahn CNIII, Ault T, Isenstein H, Potetz L, Gelder SV. Snapshot of hospital quality reporting and pay-for-performance under Medicare. Health Aff (Millwood). 2006;25(1):149-162.

12. Elixhauser A, Steiner C, Harris DR, Coffey RM. Comorbidity measures for use with administrative data. Med Care. 1998;36(1):8-27.

13. Chaudhry B, Wang J, Wu S, et al. Systematic review: impact of health information technology on quality, efficiency, and costs of medical care. Ann Intern Med. 2006;144(10):742-752.

14. Graber MA, Randles BD, Ely JW, Monnahan J. Answering clinical questions in the ED. Am J Emerg Med. 2008;26(2):144-147.

15. Bonis PA, Pickens GT, Rind DM, Foster DA. Association of a clinical knowledge support system with improved patient safety, reduced complications and shorter length of stay among Medicare beneficiaries in acute care hospitals in the United States. Int I Med Informatics. 2008;77(11):745-753.

16. Jha AK, Orav EJ, Dobson A, Book RA, Epstein AM. Measuring efficiency: the association of hospital costs and quality of care. Health Aff (Millwood). 2009;28(3):897-906.

17. Bradley EH, Herrin J, Elbel B, et al. Hospital quality for acute myocardial infarction: correlates among process measures and relationship to short-term mortality. JAMA. 2006;295.

18. Yasaitis L, Fisher ES, Skinner JS, Chandra A. Hospital quality and intensity of spending: is there an association? Health Aff (Millwood). 2009;28(4):w566-w572.

19. Jollis JG, Ancukiewicz M, DeLong ER, Pryor DB, Muhlbaier LH, Mark DB. Discordance of databases designed for claims payment versus clinical information systems. Implications for outcomes research. Ann Intern Med. 1993;119(8):844-850.

20. Losina E, Barrett J, Baron JA, Katz JN. Accuracy of Medicare claims data for rheumatologic diagnoses in total hip replacement recipients. J Clin Epidemiol. 2003;56(6):515-519.

21. Mouchawar J, Byers T, Warren M, Schluter WW. The sensitivity of Medicare billing claims data for monitoring mammography use by elderly women. Med Care Res Rev. 2004;61(1):116-127.

22. Steinwachs DM, Stuart ME, Scholle S, Starfield B, Fox MH, Weiner JP. A comparison of ambulatory Medicaid claims to medical records: a reliability assessment. Am J Med Oual. 1998;13(2):63-69.

23. Butler Nattinger A, Schapira MM, Warren JL, Earle CC. Methodological issues in the use of administrative claims data to study surveillance after cancer treatment. Med Care. 2002;40(8 suppl):IV-69-74.

24. Quam L, Ellis LB, Venus P, Clouse J, Taylor CG, Leatherman S. Using claims data for epidemiologic research. The concordance of claims-based criteria with the medical record and patient survey for identifying a hypertensive population. Med Care. 1993;31(6): 498-507.

25. Hicks J. The Potential of Claims Data to Support the Measurement of Healthcare Quality. Santa Monica, CA: Rand Corporation; 2003. 